\title{
Towards an Adaptive Learning System Based on a New Learning Object Granularity Approach
}

\author{
Amal Battou \\ IRF-SIC Laboratory, Faculty of science, \\ Ibn Zohr University \\ Agadir, Morocco \\ Chihab Cherkaoui \\ IRF-SIC Laboratory, ENCG \\ Ibn Zohr University \\ Agadir, Morocco
}

\author{
Ali El Mezouary \\ IRF-SIC Laboratory, Faculty of science \\ Ibn Zohr University \\ Agadir, Morocco \\ Driss Mammass \\ IRF-SIC Laboratory, Faculty of science \\ Ibn Zohr University \\ Agadir, Morocco
}

\begin{abstract}
To achieve the adaptability required in ALS, adaptive learning system (ALS) takes advantage of granular and reusable content. The main goal of this paper is to examine the learning object granularity issue which is directly related with Learning Object (LO) reusability and the adaptability process required in ALS. For that purpose, we present the learning objects approach and the related technologies. Then, we discuss the fine-grained as a fundamental characteristic to reach the adaptability and individualization required in ALS. After that, we present some learning object granularity approaches in the literature before presenting our granularity approach. Finally, we propose an example of implementation of our approach to test its ability to meet the properties associated with fine-grained and adaptability.
\end{abstract}

Keywords- aaptability; learning Content; adaptive learning systems, learning object; granularit;y learning content.

\section{INTRODUCTION}

The Learning Object granularity is one of the most critical properties of the Learning Object. Wiley [1] ensures that the two most important instructional properties of learning objects are reusability and granularity.

Regarding the first property, reusing LOs is believed to generate economical and pedagogical advantage over the construction of LO from scratch [2]. For the second property, researches and works on the concept of granularity in the literature are almost modest except for some works such as [3, $4,5,6,7,8]$. The most used technique is the concept of aggregation.

The relationship among reusability and granularity of learning objects is straightforward. The relationship can be depicted in a simple but effective equation: the more granular a learning object is, the more reusable it becomes [9].

As the impact of granularity is linked directly to reusability, the most difficult problem facing the designers of LO is how big a learning object should be to ensure the reusability. This problem should be at the focus of the designers of LO.
We are interested in this paper to the issue of learning content granularity and its impact on the ability to adapt, aggregate and to arrange content suiting the learner needs and preferences.

The aim of the remainder of this paper is structured as follow. We will firstly begin by "demystifying" the concept of LO and the instructional design principles. We discuss next, the granularity concept as a fundamental characteristic to achieve adaptability and individualization in the field of ALS. Afterwards, we explore the main approaches defending LO granularity from diverse angles and theirs limits. In the next section, we propose a new vision of the granularity concept and study next its ability to meet the supposed objectives of adaptability. Finally, we present the architecture of our system called ALS-CPL (Adaptive Learning System - C Programming Language) that implements the proposed approach. Then we discuss the ability of this work to achieve the adaptability required in ALS in line with other works [10, $11]$.

\section{BACKGROUND}

The Learning content is the main component of Adaptive Hypermedia System. Its development raises various issues related to the approach used. The most used one is the LO Approach.

\section{A. What is a Learning Object?}

The "Learning Object" is a new name that emerges in the field of educational resources and learning, which does not escape to ambiguity. Most proposed definitions focus on the general principles governing concept of LO such as: reusability in different situation for learning and the independence of context [12].

Balatsoukas [9] gives a typical example of the Polsani definition [8]. This author defines a LO as a unit of content Learning independent and autonomous, which is predisposed to be reuse in multiple learning contexts. Other authors such as 
Bibeau [13] considers LO as the smallest information unit or the smallest processing tool information (or applications software) used in an educational context with an intention teaching for learning through the media technology. Flamand [14] identifies three categories of LO. He distinguishes objects with little media complex and context-free (video speech of a head of state radio interview, etc.) utilitarian (modeling software, etc.) and LOs consisting of elements basic information (facts, ideas, concepts, principles, processes).

Finally, other approaches such as those of Downes [7] consider the LO size as important. Barron [5] trying to consider this approach, suggests that five to nine information objects (text, image, video, photos, etc.) can be combined to form a LO. Other works of Mortimer [6] undertaken in this direction approach the LO size in terms of time. A LO takes no longer than 15 minutes to complete.

In addition to these theoretical conceptualizations and sometimes ambiguous, other definitions emerge from various works on standardization (SCORM, LOM, IMS, etc.). For IEEE Learning Technology Standards Committee, LO are defined as any entity, digital or non-digital, which can be used, re-used or referenced during technology supported learning. Normetic [15] adds to this definition the technological support that covers the multimedia content, content instruction, educational software and software tools mentioned in a learning context to support technology. Finally, the center of Wisconsin online resources [16] defines a LO as "small learning units with a duration between 2 and 15 minutes".

The next section is designed to provide information about instructional design principles and how they relate to teaching and learning.

\section{B. The instructional design principles}

The issue of designing LO in the field of education has often been mentioned by several researchers [4, 7], etc.

Designing a LO with the features mentioned above (reusability, interoperability, durability etc.) needs to think and reflect on how to design and develop these objects. Studies of the best practices and scientific research on the LO design, reveals a series of principles and guidelines for such design [6].

We note that standards in the field of ALS, do not give steps to follow specifying methods of designing and creating LO. However, we found in the literature, a few principles that can be used in the design process and execution of LO. We will then present the most cited:

- A LO must be initially developed as "bricks" of a relatively small and designed in a way that facilitates reuse in a new educational context.

- A LO must be independent and separable from one context of use. It should contain generic information as possible.

- A LO must be indexed by metadata, based on a standard to provide information (size, author, type of interactivity, etc.) on that object. This metadata facilitates search and retrieval of LO stored in repositories.
- A LO must follow a standard format of instruction. The current standard of education facilitates the creation of uniform LOs with a clear educational strategy.

- A sequence of LOs must have a context. To build an educational unit (module, courses, etc.) from a LO, We must specify the context of (re) use of those LOs or leave the choice to learners to specify their own context.

However, even if these principles revealed some stability on the LOs design, this progress remains probably insufficient to capture the essence of the LO approach. We can cite for example, the confusion surrounding "granularity" as important attribute of LOs and which is apparent in the literature as we will present later in this paper. The granularity, as mentioned in [17], has a crucial impact on the ability to adapt, aggregate and to arrange content suiting the learner needs and preferences.

In the next section we are interested in the influence of finegrained content on the adaptability and individualization required by ALS.

\section{GRANUlARITY AND ADAPTABILITY}

The LO granularity is a key factor to allow aggregating and organizing content, to adapt the instruction to the preferences of a given learner. On one hand, an insufficient granularity (using for example large blocks of contents), probably prevents the possibility of integrating educational content in new contexts and new ALS. On the other hand, the fact of splitting up contents in several LOs of small size with a main idea, allows several options for adaptation [12].

The first possibility is to aggregate and arrange multiple objects to create other more consistent and reusable objects. The second possibility is to build and customize a LO by proposing several presentations with different computer interfaces. Another possibility implies a classification of LOs into classes of objects (for example theorems, definitions, etc.), which makes it possible to filter them more easily, improve research and thus to individualize the content.

In addition, the granularity combined with the indexing plays an important role to facilitate the adaptability. Indeed, instead of adding meta-data to big blocks of contents, learning objects of "fine granularity" are indexed, which increases thus the research space. This distinction also helps to increase the possibilities of finding the most adapted elements to a specific situation. It also allows annihilating the research silence, which can be due to an insufficient granularity.

The adaptability here then consists in choosing between the various grains those who are appropriate to a given situation.

To show the correlation between the granularity and adaptability, we propose the following learning objects granularity approach to allow a flexible representation, respecting the standards and capable of building contents in a dynamic way, from basic fragments, from the representation of the learning domain and the learner model. But before this, we study first the main used approaches of LO granularity in the literature and discuss their limits. 


\section{REVIEW OF GRANULARITY IN THE LITERATURE}

There are two main approaches defending LO granularity from two different angles.

The first one, supported by several authors like $[3,4,5,6$, $7,8]$.This approach focuses on the content. The granularity of a LO is related to the number of concept combination in this LO. A LO is called granular and therefore has great potential for reuse if it contains one basic concept.

\section{A. Approach focus on content}

For Wiley [4], the granularity of a LO depends closely on the context in which the granule will be inserted.

Similarly, South [18] defines the granularity in terms of content domain of LO which suggests that the objects have the greatest potential for reuse when they are focused on a single concept.

Other authors such as Polsani [5] argues that the granularity depends on the size of a LO. But the size designated by the author cannot be expressed in terms of bytes or duration of a LO. Size here refers to the number of ideas that a LO can transmit.

Generally, a LO must transmit one or few ideas. In the case where a LO consists of several ideas, one of these ideas may be primary and the others derive or depend directly on this one. The "fine grained" is then to considers concept as unifying principle that frees the LO of any consideration related exclusively to the size such as time or the subjectivity of the designer.

\section{B. Approach focus on media}

Concerning the second approach, it's supported by several specifications and standards bodies (IMS Global Learning Consortium, IEEE LTSC, etc.). This approach is based on a definition of granularity focused on the media. The LO granularity is directly related to the media that will be combined to create larger LOs. It uses the concept of level of aggregation instead of the notion of granularity and provides models of educational content that provide a means for defining structures.

SCORM defines an associated structure with three levels of aggregation, indicating three main components. the asset presents the smallest piece of reusable educational content that may be Web pages, animations, pictures, videos, etc, the Sharable Content Object (SCO) can be composed of several assets and the Content Organization (COs) bound to a tree structure that acts like a table of contents.

For LOM, this model consists of four levels of aggregation or "functional granularity". The first level is the lowest level of aggregation; it consists of raw media or fragments. The second level includes a collection of learning objects of level 1 , such as a lesson. A collection of LOs of level 2 such as a course constitutes the third level. The fourth level of granularity is composed by a set of courses which lead to a certificate or a diploma.

Cisco Systems has published a strategy based on the concept "RLO / RIO". The content structure is composed of two basic levels: the RIOs and the RLOs. A RIO (Reusable Information Object) is a reusable granule independent of the publishing format. It is presented by five various types of knowledge, including concepts, facts, procedures, processes or principles and associated with assessments (usually two) to evaluate the learner's assimilation of different concepts, facts, etc. A RLO (Reusable Learning Object) is the result of a combination of five to nine $(7 \pm 2)$ RIOs, attached to an overview and summary, to meet a clearly defined educational objective.

We note that other approaches are also mentioned in the literature, we cite, for example, the approach based on the execution time as a criterion of LO granularity. This approach is supported by the Wisconsin Center [19] which states that the execution time / consultation of LO must not exceed 15 minutes. Another approach is also cited in the literature that is based on the LO size in terms of bit. We believe that these two approaches are a bit outdated as it refers neither to the LO content or the presentation.

\section{Discussion}

The different approaches presented in the previous parts, tried to break down the content into a set of items or building blocks that make sense, also called grain teaching. Defined grains, although they can be reused in other contexts, probably don't fully satisfy the concept of fine grained LO that allows several option for adaptation (as we present above).

For the first approach, it defines the granularity of the LO based on the number of concept and idea combined in this LO. It does not put any restriction on the number of concept or idea or their types (complex, easy uncomplicated). Indeed, the concept of a given area may contain several ideas with different levels of complexity. This allows several other possibilities for cutting this concept on sub concepts or simple idea. Moreover, this approach does not take into consideration the presentation of a LO that can contain different format that is to say that LO can hold together text, image, video, etc. There is no restriction on either the presentation or the content of the idea presented. In our opinion this approach does not define the fine-grained as we seek to reach for better adaptability of learning content.

Regarding the second approach, in all the presented models, the first level (Asset, RIO multimedia object) cannot really match the criteria of sense unless the grain is associated with an educational objective. The size criterion is not necessarily considered and generally depends on the designer. Indeed, in most of these models there is no information about the size or the semantic density (number of idea) of o LO. In addition to the standard definition of a LO differs from one model to another. Each model is a specific profile. The LO use by one model can't be reused in another model.

\section{A NEW FORMULATION OF GRANULARITY}

Confusion surrounding granularity as an important attribute of LOs is apparent in the literature [15].

The notion of granularity, we propose, is based on several approaches of the LO granularity. In our opinion a fine grained LO is to combine the concept of meaning in terms of ideas 
carried by the grain, size in term of time of execution/consultation and media type as unifying principle. In addition, we propose a semantic structuring educational content.

For our approach, the main concept is fragment that corresponds to the notion of learning object. It can be an introduction, an assessment, exercise, a synthesis, an observation, motivation, definition, example, etc. Each of these fragments is presented by bricks multimedia: text, image, sound, video, simulation, animation, etc. A fragment has a size in terms of execution/consultation time of the corresponding brick media. The fragment size must not exceed 15 minutes. In addition, it must send a simple idea and it is described by a single brick multimedia.

The notion of fragment we use is an abstract concept. It corresponds to a multimedia brick related to an educational objectives and a semantic description.

The approach we propose is between several approaches. Indeed, it offers a functional aggregation of four main levels: courses, documents, fragments, and multimedia brick Fig. 1.

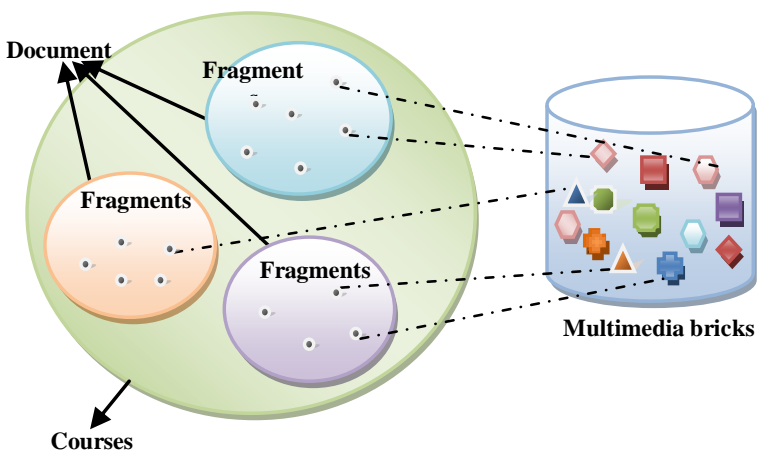

Figure 1. The proposed content model

The multimedia bricks in our approach correspond to Assets of multimedia objects and SCORM LOM, but by removing the proceeding related to a web page, which is not considered fine enough since it can present several ideas, has a large size (in term of execution time) and can contains different presentation(text, image, video, etc). The fragments in our model can be an introduction, a definition, an example, an exercise, a paragraph, a comment, an evaluation, a synthesis, or an illustration, etc. It May corresponds to the notion of RIO defined in the model CISCO.

Taking as example the fragment of the introduction, it can be presented by the text, contains one idea that is to introduce the topic of the concept. Also the time of playback time of the introduction should not exceed 15 minutes at worst (if we have a slow learner in the reading). In addition, our grains meet the test range of ideas and meaning. Indeed, the multimedia bricks are closely associated with fragments. Thus, a definition can be materialized by a picture, video, sound, etc. This increases the capacity of adaptability because the brick that will be chosen for the presentation depends on the preferences of learners. In addition, the multimedia bricks are associated with the metadata defined. The choice of a multimedia brick depends on the progress of a learner (acquired concepts, etc.), Preferences and such prior knowledge. Also linked to the semantic level that facilitates storage and improving grain research in response to a given situation.

The next part presents the implementation of our granularity approach to validate its capacity to achieve adaptability required in ALS.

\section{A CASE OF STUDY : ALS-CPL SYSTEM}

In this section we illustrate some functionalities of our system called Adaptive Learning System-the C Programming Language (ALS-CPL) which implements the LO granularity approach we proposed above.

The architectural design of the proposed system is composed by three main components Fig. 2.

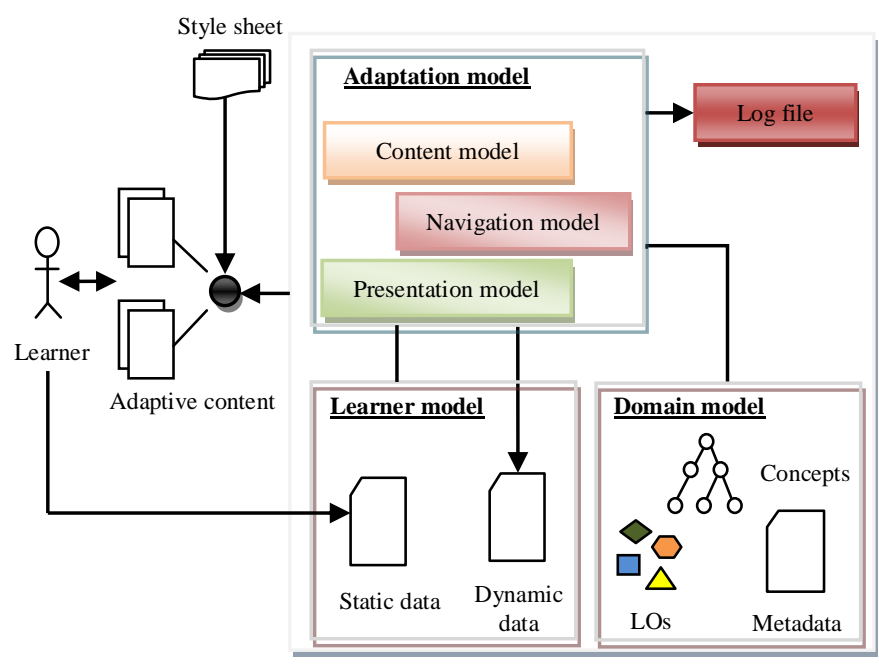

Figure 2. General architecture of ALS-CPL

From this figure we identify directly the main components of an ALS-CPL and their contents in terms of subcomponents. In the following, we present these components, their descriptions, their features and interactions between them

\section{A. The Domain model}

The domain model is characterized by its competence in terms of representation of concepts to learn, the resources available to learners and the structuring of various elements of the field

We have separated the domain model into two parts: one that includes all domain concepts that the learner can learn, regardless of the different kind resources that enable the acquisition. The second one, the most important for our work, concerns the LOs used for the acquisition of these concepts accompanied by their metadata. We create LOs according the criterions of the proposed granularity approach and the principles instructional design we present above.

This part consists of an editor of LOs Fig. 3 and reflects the semantic model presented above. This component uses PHP code to load one of the LO that we created. This editor permits the editing of the metadata to qualify LO by exploiting meta- 
data and the necessary descriptors so important to characterize each LO.

The multimedia bricks and concepts (Learning goal) can be associated to the LO. Other items for indexing LOs are added in this step. As output, of this form, an XML file is generated e.g Fig. 4.

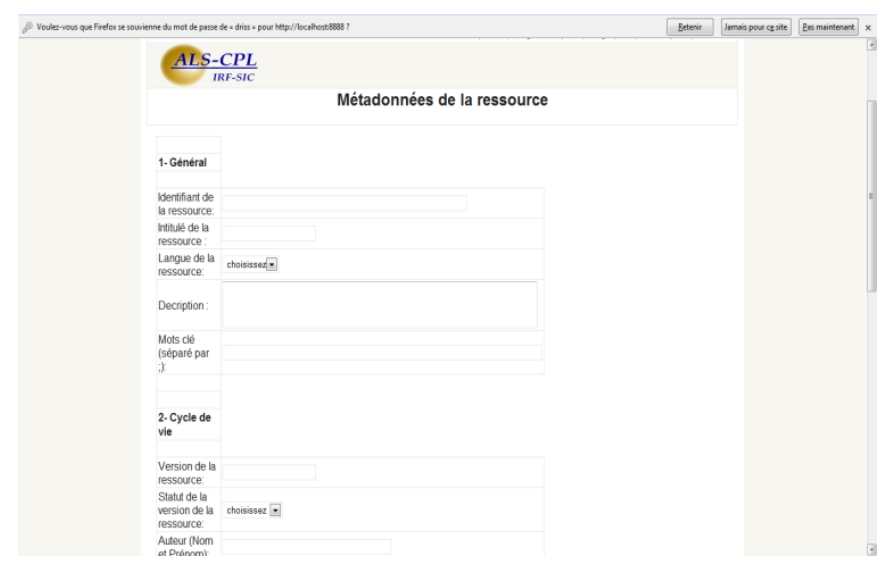

Figure 3. Editing Learning Object metadata

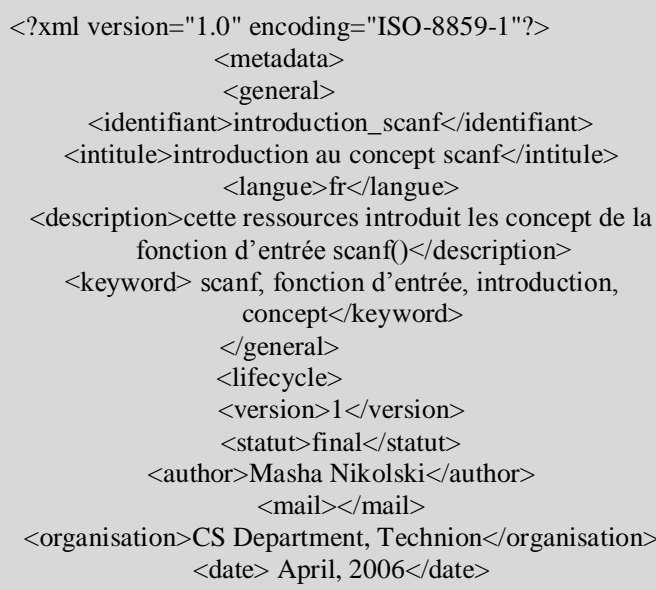

Figure 4. Example of an XML file of metadata

\section{B. The learner model}

The learner model represents the information Fig. 5 known by the system about the learner. Three strands of information are considered: personal (name, email address, phone, etc.), preferences (language preferences, favorite colors, the preferred type of educational content, etc.) and Knowledge of learner that is described in relation to each domain model. This component evolves dynamically as the student progress in his course.

The learner model we make is open for editing and viewing by both the learners themselves and the system. The aim of this choice is to involve the learner in the construction of his model. So that it contains information and makes it more reliable and more representative of the learner.

For our model, it consists of two main parts:
- Static data: the data is indicated by the student during his first access to the system and can be updated by the learner at any time of his learning.

- Dynamic Data: This data is updated only by the system and highly dependent on the results and interactions of the learner with the content presented.

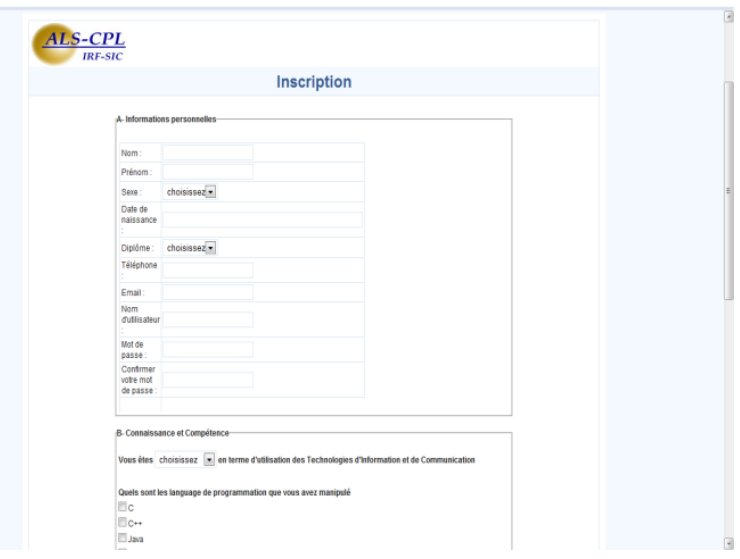

Figure 5. Editing the learner model information in ALS-CPL

As output, an XML file is generated. Fig. 6 presents an example of a model learner.

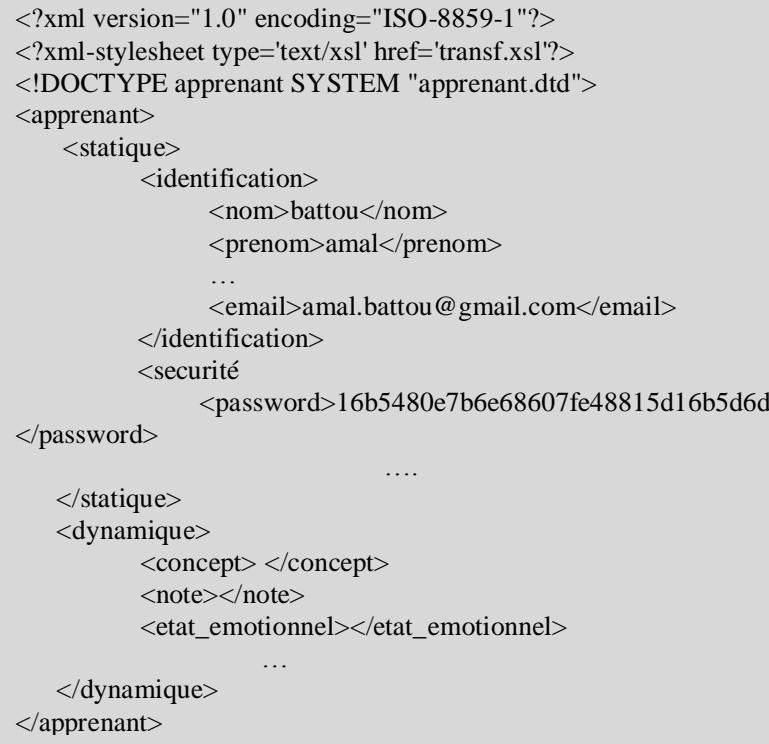

Figure 6. Example of an XML file of a learner model

\section{The adaptation model}

The adaptation model deals with the generation of adaptive content that will be subsequently presented to the learner. This component has three sub-component: the navigation model, the presentation model and the content model. Each subcomponent contains a set of rules to achieve the adaptation.

- The model navigation: the navigation model defines the structure of the hypermedia system and describes how to traverse the various nodes of the system. 
- The presentation model: it is used to adapt the layout for the visual line with the preferences or needs of the learner.

- The content model: this model is used to provide additional content, similar content, alternative content, or hide content.

The process of how these sub-components Fig. 7 and an example of a content interface Fig. 8 are presented as follow.

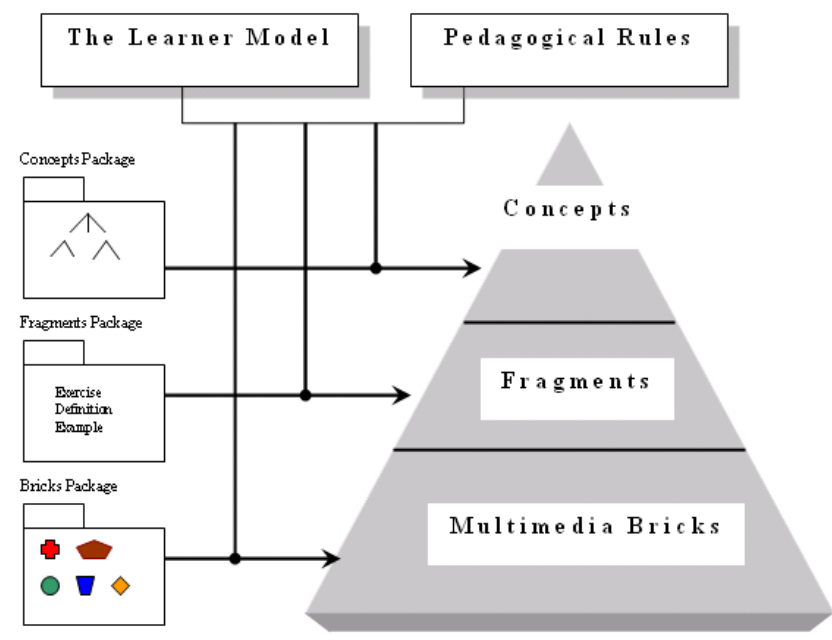

Figure 7. The assembling courses process.

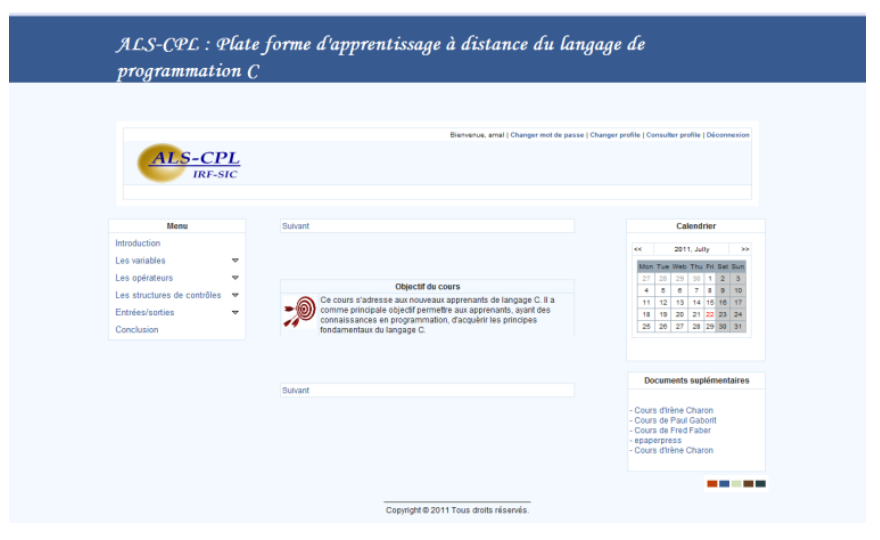

Figure 8. Example of a content interface

The simplest case, when a learner interacts for the first time with the system, the list of the acquired concepts is empty. The concepts that have no pre-requisites in the graph of the concepts and have not been acquired will initialize the list of the active concepts, which enable to choose the objective of the session.

Some elements of the learner model can influence this decision. These considerations come from the background knowledge and skills of the learner represented in the learner model like the level concerning the programming languages (beginner, intermediate, Expert), or the background knowledge composed of a set of programming concepts (variables, decision-making code, loop structures, procedures and functions, data bases, etc.). Some pedagogical rules for such decision are applied.

The choice of one or more concept(s) associated with other information coming in particular from representations of the learner, determines a sequence that will then be derived in fragments. If, for example, the model of the learner indicates that he (she) prefers to learn by examples, the sequence will consist of more examples. For exercises, the difficulty level will depend on information extracted from the model of the learner corresponding to his level (Beginner, Intermediate, and Advanced).

This sequence corresponds to a prototypical sequence of fragments to achieve the selected learning concept. For each fragment of this sequence, the system associates a multimedia brick, still according to the learner model. If the learner model indicates for example that learner prefer pictures and videos, the system will promote anything that is multimedia. If he (she) prefers reading on the screen, the text associated with fragments will be used to create a course document.

\section{DISCUSSION}

The fine granularity concept that we have presented above, meets the criterion of scope ideas and meaning. Indeed, the bricks are closely associated with fragments. Thus, a definition can be materialized by a picture, video, sound, etc. This increases the capacity of adaptability because the choice of bricks depends on the learners preferences. We note that each fragment corresponds to a single brick multimedia depending on the preference of the learner (video, audio, text, etc.)

In addition, the bricks are associated with domain concepts through metadata set. The choice of a brick depends on the progress of a learner (acquired concepts, etc.), preferences and those acquired earlier. Also, the semantic level linked to the indexing, allows easy storage and improving grain research responding to a given situation.

Finally, we can highlight some correlation of our model compared with recent work focusing on adaptability in dynamic adaptive hypermedia. We emphasize in particular the work on the project Medyna [10] and work related to the assembly of existing resources by using graphs and operators of decision [11]. Our model is also inspired by the work of Brusilovsky [20] on the graphs of concepts and the relationship between concepts, brick fragments and multi-media, with a distinction related to meta-data used and techniques for adaptability and assembly course.

\section{CONCLUSION}

In this paper, we have proposed a model of granular LO for the adaptability and the re-use of the learning contents. This model of content is designed respecting to the various characteristics of the stated granularity. The first advantage of this model is its hierarchical structure in the form of "grains" of contents which respect the specifications of the existing standards (LOM, SCORM, etc.). Another advantage lies in the fact that the same fragment or a multimedia brick could easily be re-used in several documents or then directly in another context of learning. We can also note that the model suggested 
is open. It can indeed employs the proprietary format of the contents, or import it from the web. Moreover, the granularity combined with indexing plays an important role in facilitating the search mechanism and adaptability. Indeed, instead of adding metadata to large blocks of educational content, small size granules are indexed, which enlarges the search space.

As a second point, we have showed the system architecture able of integrating the LOs infrastructure, the domain concept structure and the learner model interface. Different interfaces are presented.

It is clear that several issues remain to be addressed to arrive at the expected system. Our work continues along these lines to try to finish a first functional prototype which will be tested and validated.

\section{REFERENCES}

[1] D.A. Wiley, "Learning Objects and the New CAI: So what do I do with a learning object?", $1999 . \quad$ Retrieved in http://opencontent.org/docs/instruct-arch.pdf.

[2] X. Ochoa, E. Duval, Measuring Learning Object Reuse In Times of Convergence. Technologies Across Learning Contexts, Vol. 5192, 2008, pp. 322-325.

[3] P. Balatsoukas, A. Morris, A. \& O’Brien, "Learning Objects Update: Review and Critical Approach to Content Aggregation", Educational Technology \& Society, 2008, Vol. 11 (2), pp-119-130.

[4] D.A. Wiley, "Connecting learning objects to instructional design theory: A definition, a metaphor, and a taxonomy". In D.A. Wiley (Ed.), The instructional use of learning objects, 2000.

[5] P.R. Polsani, "Use and abuse of reusable learning objects", Journal of Digital Information, 2003, 3(4). Retrieved in http://jodi.tamu.edu/Articles/v03/i04/Polsani/.

[6] S. Downes, "Design and reusability of learning objects in an academic context: A new economy of education", JSDLA Journal, 2003, 17(1). Retrieved http://www.usdla.org/html/journal/JAN03_Issue/article01.html.

[7] T. Barron, "Learning object approach is making inroads", In Learning Circuits, 2002. Retrieved in http://www.learningcircuits.org/2002/may2002/barron.html.

[8] L. Mortimer, "Learning objects of desire: Promise and practicality". Learning Circuits, 2008, Retrieved In http://www.astd.org/LC/2002/0402_mortimer.htm.

[9] I.F. Silveira, "Reusability and Interoperability of Adaptive Learning Objects Repositories" Symposium de l'informatique en education, 2005. Retrieved in http://www.brie.org/pub/index.php/sbie/article/viewFile/407/393.

[10] A. Behaz, "Création dynamique de documents hypermédias adaptatifs", Available: http://lodel.irevues.inist.fr/cide/index.php?id=281.

[11] A. Bouzeghoub, "Un modèle de description sémantique de ressources pédagogiques basé sur une ontologie de domaine", Sciences et Technologies de 1'Information et de la Communication pour l'Éducation et la Formation, Vol. 12, (2005).

[12] A. Battou, A. El Mezouary, C. Cherkaoui, D. Mammass, The granularity approach of learning objects to support adaptability in adaptive learning systems. Journal of Theoretical and Applied Information Technology.

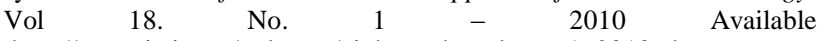
:http://www.jatit.org/volumes/eighteenth_volume_1_2010.php.
[13] R. Bibeau, "Les TIC à l'école : proposition de taxonomie et analyse des obstacles à leur intégration", EpiNet n ${ }^{\circ} 79$, novembre 2005. Available : http://www.epi.asso.fr/revue/articles/a0511a.htm.

[14] P. Flamand, A. Gervais, "Les objets d'apprentissage : au-delà de la technologie, la pédagogie", Le bulletin Clic, 2004, N54, pp 1-5. Available : http://www.clic.ntic.org/clic54/objets.html.

[15] Normetic, "La description normalisée des ressources : vers un patrimoine éducatif', CREPUQ Novasys Inc., 2003. Available : http://www.profetic.org:16080/normetic/pdf/normetic.pdf.

[16] WISC, (2009) "Visconsin on line Ressource center". Available : http://www.wisconline.com.

[17] M. Memmel, E. Ras, P.Klaus. J. and M. Yacci. "Approaches to learning Object Oriented Instructional Design", Learning Objects and Instructional Design, pages 281-326. Informing Science Press, 2007, Retrieved in http://www.dfki.unikl.de/ memmel/literature/2007/MemmelRasJantkeYacci+07.pdf.

[18] J.B. South, D.W. Monson, (2000). A university-wide system for creating, capturing, and delivering learning objects. In D. A. Wiley (Ed.), The Instructional Use of Learning Objects [Online] Available : http://reusability.org/read/chapters/south.doc.

[19] WISC, (2009) "Visconsin on line Ressource center". Available : http://www.wisconline.com.

[20] P. Brusilovsky, "Adaptive Hypermedia: From Intelligent Tutoring Systems to Web-Based Education", 2000, Available: http://www2.sis.pitt.edu/ peterb/papers/ITS00inv.html.

\section{AUTHORS PROFILE}

Mrs A. Battou received her Master's degree in Applied Mathematic and Computers in 2008 in the university Ibn Zohr, Faculty of Science Agadir. Morocco. She is pursuing her $\mathrm{Ph}$. D degree. Her doctorate study is interested by adaptability in Adaptive Learning Systems, using Learning Objects. She is currently a state engineer obtained from a high school of of Engineering and works at the University Ibn Zohr since five years.

Mr A. EL Mezouary received his Master's degree in Applied Mathematic and Computers in 2008 from the university Ibn Zohr, Faculty of Science Agadir. Morocco. He is a research student pursuing his Ph. D degree. His thesis is part of a general problem of the Educational Semantic Web. He is interested specifically by the adaptability in Adaptive Learning Systems, using Learning Objects and the Semantic Web. He served as professor of computers and data processing at the secondary school.

Dr. C. Cherkaoui is currently research professor at the School of Business and Management Agadir - Morocco. He holds a doctorate in computers and data processing at a French university since 1996. The research area is part of continued discipline of Artificial Intelligence. He has worked on various topics such as: planning and action theory, adaptive systems for human learning, multi-agent systems, e-learning, knowledge engineering, knowledge representation including ontologies. He obtained his habilitation to direct research in 2006. He has published various articles and papers in journals and international conferences. He served as Head of Department of Computer Science and is currently team leader of the research group of Intelligent and Communicating Systems.

Dr. D. Mammass is Professor of higher education at the Faculty of Science of Agadir since a score of years. He obtained a state doctorate in Mathematics and Image Processing in 1999. He currently directs several theses in varied disciplines of mathematics and data processing such as: the geographical Information Systems, the Digital Processing of the images and pattern recognitions, the geographical databases, the knowledge management, the semantic Web, etc. He is currently Director of the School of Technology Agadir and head of the laboratory IRF-CLC (Image Recognition of Forms, Intelligent Systems and communicating). 